\title{
Effects of lifestyle advice provided by pharmacists on blood pressure: The COMmunity Pharmacists ASSist for Blood Pressure (COMPASS-BP) randomized trial
}

\author{
Hiroshi Okada ${ }^{1,2,3}$, Mitsuko Onda ${ }^{4}$, Masaki Shoji ${ }^{5}$, Naoki Sakane ${ }^{2}$, Yasushi Nakagawa ${ }^{6}$, \\ Takashi Sozu ${ }^{7}$, Yui Kitajima ${ }^{8}$, Ross T. Tsuyuki ${ }^{3}$, Takeo Nakayama ${ }^{1}$ \\ ${ }^{1}$ Department of Health Informatics, Kyoto University School of Public Health, Kyoto, Japan; \\ ${ }^{2}$ Division of Preventive Medicine, Clinical Research, National Hospital Organization, Kyoto Medical Center, Kyoto, Japan; \\ ${ }^{3}$ EPICORE Centre, Department of Medicine, Faculty of Medicine and Dentistry, University of Alberta, Edmonton, Alberta, Canada; \\ ${ }^{4}$ Clinical Laboratory of Social and Administrative Pharmacy, Osaka University of Pharmaceutical Sciences, Osaka, Japan; \\ ${ }^{5}$ Clinical Laboratory of Practical Pharmacy, Osaka University of Pharmaceutical Sciences, Osaka, Japan; \\ ${ }^{6}$ Polon Company, Kobe, Japan; \\ ${ }^{7}$ Department of Information and Computer Technology, Faculty of Engineering, Tokyo University of Science, Tokyo, Japan; \\ ${ }^{8}$ Department of Management Science, Faculty of Engineering, Tokyo University of Science, Tokyo, Japan.
}

\begin{abstract}
Summary The COMmunity Pharamcists ASSist for Blood Pressure (COMPASS-BP) study aimed to assess the effectiveness of lifestyle support programs administered in community pharmacies on hypertension control. This open-label, two-armed parallel group, cluster-randomized controlled trial included 73 pharmacies (clusters) in Japan randomized to a control or intervention group. Eligible hypertensive patients $(n=125)$, aged 20-75 years, received the intervention $(n=64)$ or the control treatment $(n=61)$, as dictated by their pharmacy randomization. Patients in the intervention group received brochures and healthy lifestyle advice from pharmacists using motivational interviewing methods during pharmacy visits over a 12-week period, with their usual pharmacy care. Conversely, the control group just received usual care. The main outcome measure was a change in morning systolic blood pressure (SBP) from baseline to week 12. The intervention group exhibited a decrease in morning SBP that was $6.0 \mathrm{mmHg}$ greater than that of the control group $(95 \%$ confidence interval $[C I]:-11.0$ to $-\mathbf{0 . 9}, p=\mathbf{0 . 0 2 1})$. In a mixed-effect model for repeated measures analysis, the intergroup difference in morning SBP decrease was $-4.5 \mathrm{mmHg}(95 \% \mathrm{CI}:-8.5$ to $-0.6, p=$ 0.024). Our findings indicate that implementation of a lifestyle advice program in pharmacies is feasible and may lead to reduced blood pressure.
\end{abstract}

Keywords: Community pharmacy, hypertension, home blood pressure measurement, patient education, mixed-effect model for repeated measures analysis

\section{Introduction}

One of the most common and important health problems faced by societies worldwide is hypertension. In particular, elevated morning systolic blood

Released online in J-STAGE as advance publication December $18,2017$.

*Address correspondence to:

Dr. Hiroshi Okada, EPICORE Centre, Department of Medicine, 362 Heritage Medical Research Centre, University of Alberta, Edmonton AB, Canada T6G2S2.

E-mail: hiroshi@ualberta.ca pressure (SBP) has been identified as a risk factor for atherosclerotic disease $(1,2)$, which is responsible for at least $45 \%$ and $51 \%$ of heart disease- and strokerelated deaths, respectively (3). Although these vascular diseases are major causes of death in Japan, only about $30 \%$ of men and $40 \%$ of women have controlled blood pressure (BP) (4). Home BP monitoring is preferred over hospital, clinic, or office BP measurements $(5,6)$. Home BP monitors are sold in most community pharmacies in Japan. An estimated $77 \%$ of individuals with hypertension and $40 \%$ of individuals without hypertension use these devices (7).

Lifestyle plays an important role in the management 
of hypertension. The provision of lifestyle advice was found effective in decreasing the recipient's weight, dietary sodium intake, and alcohol intake, while promoting physical activity, and resulted in better BP control (8-11). In Japan, the estimated average daily sodium intake is $10.6 \mathrm{~g}$ per day, which is more than twice the amount recommended by the World Health Organization $(3,7,9)$. However, individuals with hypertension have very few opportunities to learn about healthy lifestyles from professionals in their communities.

Pharmacists are highly accessible healthcare professionals who can be a valuable social asset in the management of hypertension (3). Previous studies have shown that the administration of a care-based program to hypertensive patients at community pharmacies improved patients' BP control and outcomes (12-14). However, the consultations provided in previous studies were of relatively long durations (20-30 minutes), and require a more advanced scope of practice than is available in Japan currently. Previous studies also measured BP during clinic visits, rather than using the preferred strategy of home BP measurements. Therefore, our study was aimed to evaluate the effects of brief motivational lifestyle advice provided by pharmacists to hypertensive patients with the intent to improve homemonitored BP in a community setting (COMmunity Pharmacists ASSist for Blood Pressure [COMPASS-BP] Project).

\section{Materials and Methods}

\subsection{Trial design}

This study was a cluster randomized controlled trial. The clusters were community pharmacies. The pharmacies were randomly assigned to either the Intervention group or the Control group. Randomization was stratified by matched cluster sizes. Community pharmacies were recruited through direct requests to local pharmaceutical associations and chain pharmacies during explanatory meetings. Seventy-three pharmacies in 15 prefectures across Japan participated. Pharmacists and patients were not blinded to treatment allocation. The trial registration number is UMIN000014128 (May 31, 2014) (https://upload.umin.ac.jp/cgi-open-bin/ctr_e/ctr_view. cgi? recptno $=$ R000016452)

\subsection{Participants}

Pharmacy eligibility criteria were as follows: $i$ ) more than 20 hypertensive patients visited the pharmacy each month; and ii) certified pharmacists delivered the training program according to the protocol. The protocol provided instructions on the correct method for home BP measurement through a brochure, as well as supportive communication and clinical practice guidelines regarding hypertension in Japan.

Patient eligibility criteria were as follows: $i$ ) hypertensive patients who visited pharmacies to obtain medication for more than three months; and ii) aged 2075 years.

Patient exclusion criteria were as follows: $i$ ) serious complications, such as dialysis or chronic kidney disease; ii) dementia or mental illness; or iii) an otherwise determined lack of suitability for this lifestyle intervention study.

Data collection settings and locations: Study recruitment occurred during two periods: from September 2014 to March 2015 and from May 2015 to November 2015. Pharmacists recruited patients using standardized brochures and posters. Each pharmacy was asked to recruit three patients. Eligibility was verified using the most recent clinical BP data recorded in a BP diary within the previous three months and the medication history obtained from the pharmacy.

\subsection{Randomization}

Blocked randomization according to the pharmacy size (classified by the number of patients per day: small, 0-50 patients; middle, 51-100 patients; large, $\geq 101$ patients) and type (chain or private pharmacy) was performed by the Center of Randomization at the Division of Prevention Medicine of Clinical Research Institute, National Hospital Organization Kyoto Medical Center, Japan.

\subsection{Blinding}

The participants and community pharmacists involved in this study could not be blinded because of the nature of the intervention. A statistician who was blinded to group allocation analysed the data.

\subsection{Intervention group}

\subsubsection{Pharmacist training}

Pharmacists who participated in this study completed a 4-hour training program on the methods for interviewing patients and providing information at community pharmacies. Candidate pharmacists were trained in the following three areas: $i$ ) The study purpose, ii) Correct home device methods for BP measurements, and iii) The provision of brief motivational advice to patients. The modified motivational interviewing, which was based on empowerment or coaching-style communication included three steps: $i$ ) using an open question, $i i$ ) setting each goal with patients, and iii) closing with encouragement. The program had the following five areas that could be selected by patients: $i$ ) reducing sodium intake, $i i)$ including more vegetables in meals, iii) exercising, $i v$ ) losing weight, and $v$ ) reducing alcohol consumption. 


\subsubsection{Patient training and registration}

Validated home BP monitors (HEN-7200; Omron Corporation, Kyoto, Japan) (15) and pedometers (HJ205; Omron Corporation) were provided to all participants upon study entry at the pharmacy. The participants were instructed how to measure BP and count steps, then collected these data for two weeks before study randomization. The pharmacists checked these BP data for the initial two weeks; patients with less than two missing measurements were registered in the study (Figure 1).

Intervention group patients submitted their BP measurements and other personal data for evaluation and received advice about a healthy lifestyle from a certified pharmacist when they visited the pharmacy. The attending pharmacists were provided with 12 types of brochures, a BP diary, and a table listing food with high sodium content to support the five previously mentioned agenda items. The pharmacists gave the participants one of these tools and demonstrated its usage.

At the second visit (visit 1), a pharmacist and the Intervention group patient discussed goal setting and methods to induce lifestyle changes, according to the five agenda items. Pharmacists provided this information in a brochure, which was used by the patient when selecting the method. At every visit (visits 1-3), the pharmacist asked about the patient's lifestyle, goal accomplishments, and barriers to lifestyle changes. After reflecting on the previous visit, they discussed new goal setting during every visit. The pharmacists delivered a standardized intervention using a check list in the Case Report Form.

\subsection{Control Group}

Patients in the Control group were provided the same home BP monitor as the Intervention group and a basic explanation about their medication. The attending pharmacists checked and evaluated their BP and measurement methods without providing special consultations.

\subsection{Outcomes}

All participants were followed for 12 weeks. The main outcome was the change in morning home SBP, which was calculated as the difference between BP data from the pharmacy at baseline (visit 0 ) and the average home $\mathrm{BP}$ at 12 weeks (78-84 days). The secondary outcomes were medication changes, medication adherence (using the Modified Morisky Scale 8-item response option) (16), lifestyle changes (using the International Physical Activity Questionnaires) $(17,18)$, changes in attitudes and knowledge about hypertension, and changes in homemonitored body mass index (BMI; weight in $\mathrm{kg} / \mathrm{squared}$ height in $\mathrm{m}^{2}$ ) between baseline (visit 0 ) and the last 12 weeks. Salt intake was determined using a validated seven-item questionnaire about the intake frequency of salty and processed foods, such as salty fish, pickles, and soups (19). Patients' quality of life was determined using the EuroQol questionnaire (20). The participants responded to the above-mentioned questionnaires at community pharmacies during their visit 0 (baseline) and visit 4 (12 weeks later). All participants wore a pedometer for more than 12 hours per day.

\subsection{Measurements}

Baseline BP was measured and recorded at the pharmacy upon study entry. In Japan, a patient diary is typically recommended to record home BP measurements and is

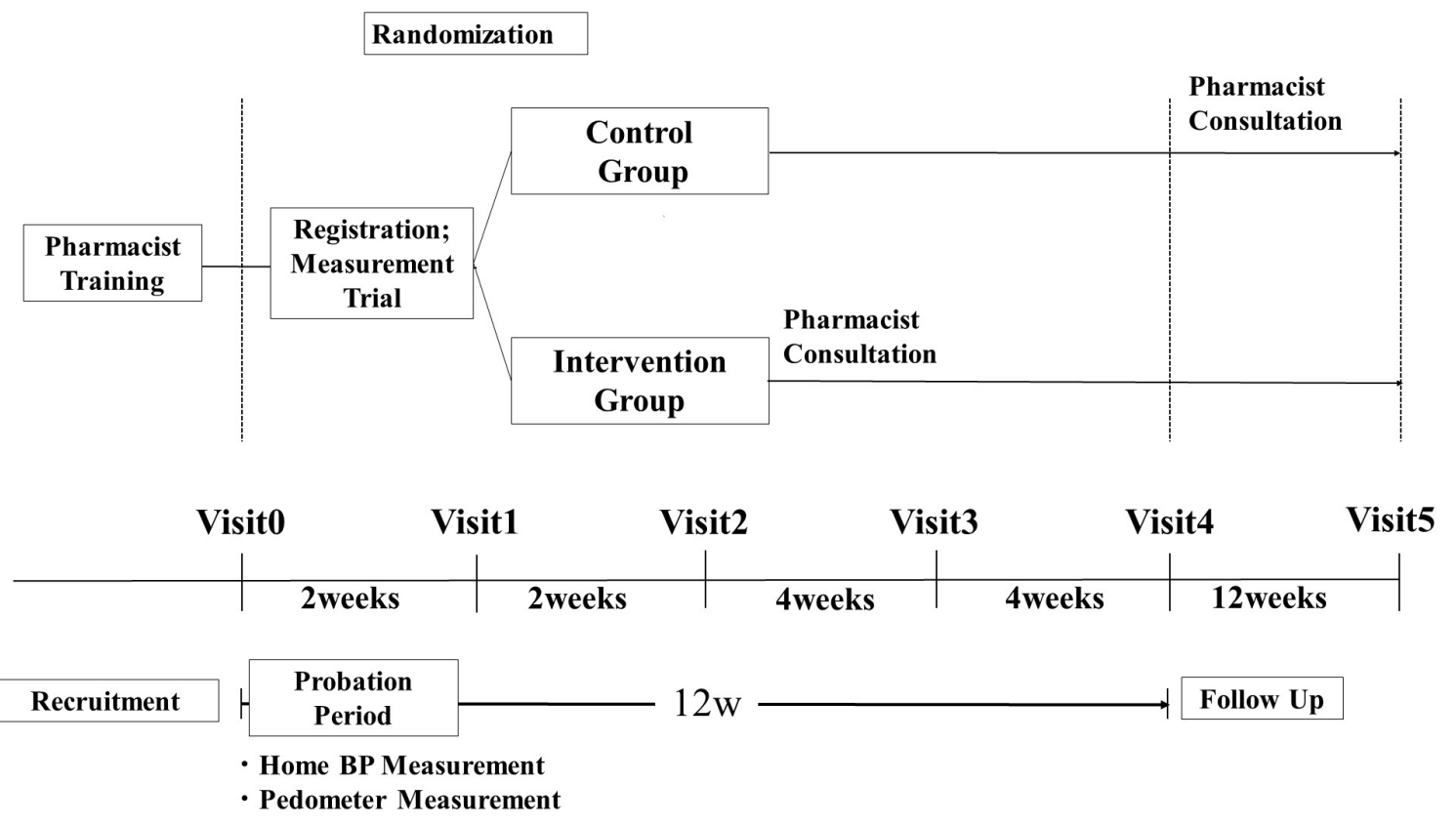

Figure 1. Study flow chart of COMPASS-BP. 
used by hospitals and clinics to evaluate home BP data for hypertensive patients. Diaries were provided to all patients in the study to record BP and additional data, such as body weight and steps taken on each monitored date. The participants measured their BP twice daily (in the morning and at bedtime) by recording this information in their diary, thus enabling the sharing of these data with physicians and pharmacists. The participants' heights and weights were also checked. Medication data were collected from prescriptions. Study data were collected from all participants for 84 days. All conditions except the designated intervention were equal between the two groups.

Securing reliability of the data: During every visit to the pharmacy, patients were educated about appropriate BP measurement procedures based on the current clinical practice guidelines in Japan (5). The attending pharmacists recorded these data. Changes in patients' medications were checked and printed for inclusion in personal health records. At every visit, the attending pharmacist was required to check both the BP measurement method and any missing records.

\subsection{Sample size}

We assumed an SBP decrease of $5 \mathrm{mmHg}$, significance level of $5 \%$, outcome variable standard deviation (SD) of 15 , statistical power of $80 \%$, and two-tailed significance test to calculate the required sample size in each group. Using an intra-class correlation of 0.1 and assuming three patients in each cluster, a minimum required sample size of 30 pharmacies per arm was determined, for 180 patients in both arms. To compensate for dropouts, the sample size was set to 33 pharmacies (99 patients) in both groups (total $=198$ ). These calculations were conducted using sample size tables for clinical studies (21).

\subsection{Statistical analysis}

Continuous variables are presented as means and standard deviations (SD), whereas categorical variables are presented as numbers and percentages. The difference in the primary outcome between the groups was estimated with $95 \%$ confidence interval and compared using Student's $t$-tests. Furthermore, repeatedly measured home BP data were analysed using the mixedeffect model for repeated measures (MMRM) with the first-order autoregressive structure (AR[1]) (22), where the group, the measurement time, and the interaction of group and measurement time were included as the fixed effect and the baseline SBP as the covariate. Secondary outcome analysis between the two groups was performed using Student's $t$-test. All $p$-values were two-sided, with $p$-values $<0.05$ considered as statistically significant. All data analyses were conducted using SAS statistical software version 9.4 (SAS Institute Inc., Cary, NC,
USA). The clustering effect was not considered because of the small cluster size (average 2 patients a pharmacy: 1-3 patients). All data are presented as means with SDs. The primary analyses used the last observation carried forward method to address missing data. For cases with missing data at baseline, we used the average BP from the first week.

\subsection{Ethics approval}

All related procedures in this study were approved by the Ethical Committee of Kyoto University School of Medicine (C1230). All patients provided written informed consent to participate in the study. The study protocol complied with the principles of the Declaration of Helsinki and written informed consent was obtained from participants prior to study recruitment. All data were analysed anonymously.

\section{Results}

Enrolment in the COMPASS-BP study began on September 1, 2014 with 55 pharmacies (112 patients) and on May 1, 2015 with another 18 pharmacies (13 patients). A total of 73 pharmacies were recruited for the study and were randomized. However, 17 pharmacies did not recruit any patients ( 7 pharmacies assigned to Intervention and 10 assigned to the Control). A total of 125 patients were recruited (64 in the Intervention and 61 in the Control). The cluster exclusion rate was $25.5 \%$ (25/98 pharmacies), and the allocated cluster rate was $76.7 \%$ (56/73 pharmacies). The final analysis included 30 pharmacies (64 patients) in the Intervention group and 26 pharmacies (61 patients) in the Control group (Figure $2)$. Although follow-up data were available for all participants, the baseline BPs for 44 of the 125 patients were not recorded; in these cases, the first week's BP was used as the baseline value.

Participants in the Intervention group were slightly younger (Intervention: 61.6 [9.9] years, Control: 66.6 [9.0]), had a lower incidence of diabetes (Intervention: 17.2\%, Control: $24.6 \%$ ), a higher incidence of hyperlipidaemia (Intervention: $37.5 \%$, Control: $29.6 \%$ ), and lower frequency of angiotensin receptorII blocker/angiotensin-converting enzyme inhibitor use (Intervention: 65.6\%, Control: 78.7\%) (Table 1). Furthermore, the mean morning SBP and diastolic blood pressure (DBP) were slightly higher in the Intervention Group (Intervention: 135.2/81.6 [13.1/8.7] $\mathrm{mmHg}$, Control: 131.8/76.6 [14.1/9.6]) (Table 2).

There were significant differences in the mean change in morning SBP and DBP between the two groups. The difference in change in SBP over the 12 weeks was -6.0 mmHg (Intervention: $-1.1 \mathrm{mmHg}$, Control: $+4.9 \mathrm{mmHg}$; $95 \%$ confidence interval $[\mathrm{CI}]:-11.0$ to $-0.9 ; p=0.021$; Table 2). The subsequent MMRM analysis yielded a slightly smaller but still significant estimated difference 


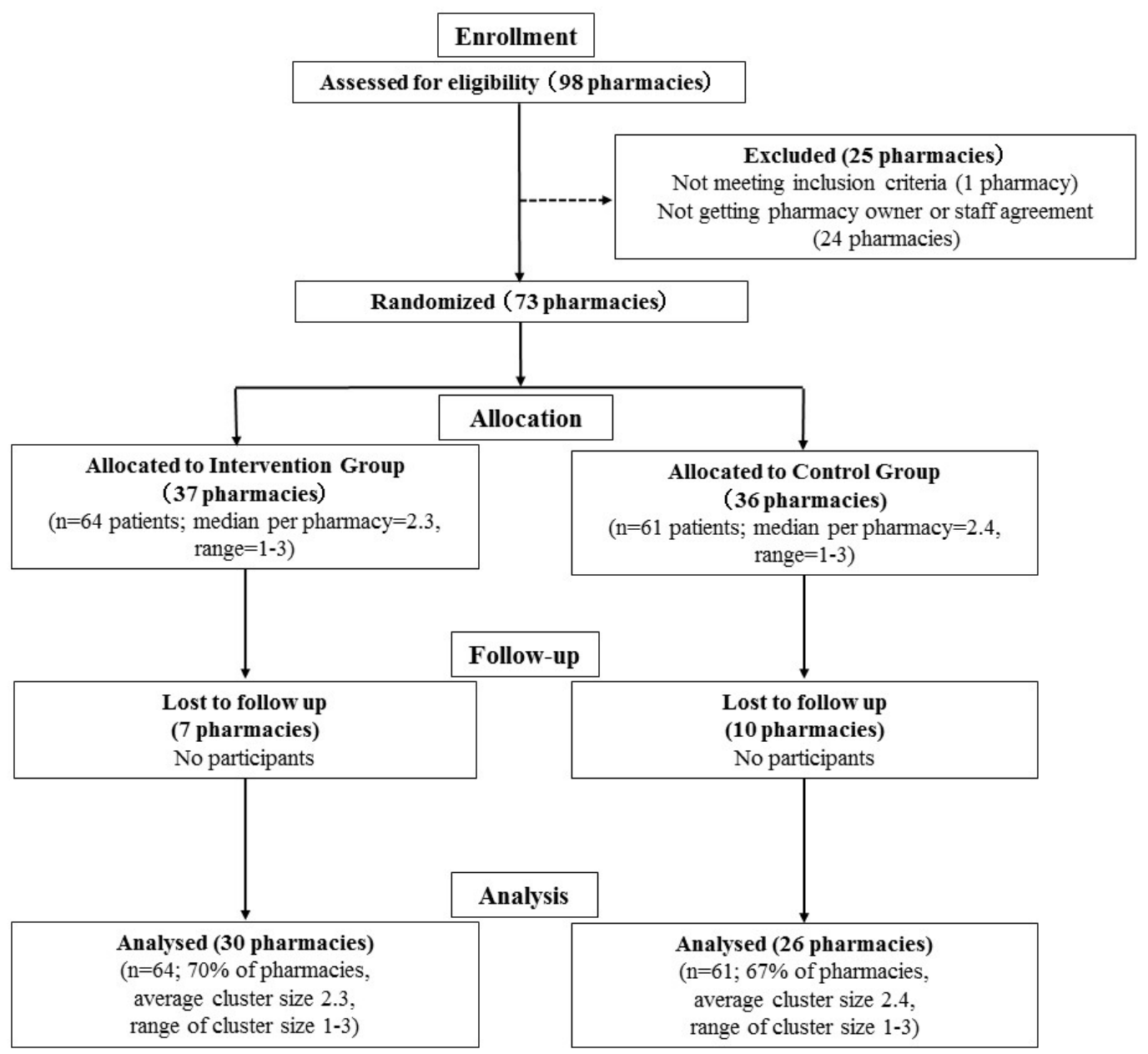

Figure 2. CONSORT flow chart of COMPASS-BP.

Table 1. Baseline characteristics of individuals and clusters

\begin{tabular}{|c|c|c|}
\hline Variables & Intervention Group & Control Group \\
\hline \multicolumn{3}{|l|}{ Cluster level } \\
\hline No. of Pharmacies & 37 & 36 \\
\hline \multicolumn{3}{|l|}{ Cluster type $(\%)^{\mathrm{a}}$} \\
\hline Small & $23(62.2)$ & $25(69.4)$ \\
\hline Medium & $7(18.9)$ & $6(16.7)$ \\
\hline Large & $7(18.9)$ & $5(13.9)$ \\
\hline \multicolumn{3}{|l|}{ Individual level } \\
\hline No. of patients & 64 & 61 \\
\hline Age, years $(\mathrm{SD})^{*}$ & $61.6(9.9)$ & $66.6(9.0)$ \\
\hline Gender, female (\%) & $40(62.5)$ & $35(57.4)$ \\
\hline \multicolumn{3}{|c|}{ Duration of Antihypertensive Medication } \\
\hline \multicolumn{3}{|l|}{ Years, $(\%)^{\mathrm{b}}$} \\
\hline$<5$ & $19 / 40(47.5)$ & 19/36 (52.8) \\
\hline $6-10$ & $8(20.0)$ & $10(27.8)$ \\
\hline $11-15$ & $7(17.5)$ & $2(5.6)$ \\
\hline $16-20$ & $5(12.5)$ & $3(8.3)$ \\
\hline$>21$ & $1(2.5)$ & $2(5.6)$ \\
\hline \multicolumn{3}{|l|}{ Comorbidities } \\
\hline Diabetes (\%) & $11 / 64(17.2)$ & $15 / 61(24.6)$ \\
\hline Hyperlipidemia (\%) & $24 / 64(37.5)$ & 18/61 (29.6) \\
\hline \multicolumn{3}{|l|}{ Medication $(\%)^{c}$} \\
\hline $\mathrm{ARB} / \mathrm{ACE}$ & $42 / 64(65.6)$ & 48/61 (78.7) \\
\hline $\mathrm{CCB}$ & $39 / 64(60.9)$ & $45 / 61(73.8)$ \\
\hline Diuretic & 11/64 (17.2) & 6/61 (9.8) \\
\hline$\alpha / \beta$-Blocker & $2 / 64(3.1)$ & 8/61 (13.1) \\
\hline Renin inhibitor & $0 / 64(0.0)$ & $1 / 61(1.6)$ \\
\hline
\end{tabular}

"Age: $p=0.004$. There were no significant differences expect for age in two groups. Missing data was not included in the analysis. ${ }^{\text {a. Pharmacies }}$ were classified by the number of patients seen per day. Small: 0-50, Middle: 51-100, Large: > 101. ${ }^{b}$ Duration of medication use for hypertension was checked through patient self-reporting. ${ }^{c}$ Categories of hypertensive medications taken by patients. 
in the changes in morning SBP between the two groups $(-4.5 \mathrm{mmHg}$; 95\% CI: -8.5 to $-0.6, p=0.024$; Table 3$)$. DBP did not differ significantly between the two groups ( $-1.8 \mathrm{mmHg}$; 95\% CI: 4.4 to 0.8, $p=0.169$; Table 3 ).

According to International Physical Activity Questionnaires scores, the groups differed in terms of changes in physical activity. More Intervention patients were less physically active (266 to 243 ; Intervention: -23 [105]; Control: 119 [191]; $p=0.016$; Table 4). No significant differences were observed for any other secondary endpoints, including quality of life, BMI, salt intake score, or knowledge about a healthy lifestyle.

\section{Discussion}

Lifestyle plays an important role in the management of hypertension. The COMPASS-BP trial was conducted to determine whether a brief, motivational interview-based intervention administered by pharmacists could improve BP. Our study demonstrated that the intervention was feasible and well accepted by pharmacists and patients. Notably, the attained SBP level was similar to those reported by previous studies $(13,14)$. Although we did not observe large differences in BP, the results implied that the brief healthy lifestyle advice provided by pharmacists during daily practice had an effect on patients' BP control.

Motivational interviewing is a patient-centred strategy designed to elicit behaviour change by helping clients to explore and resolve ambivalence to change (23). Motivational interview-based interventions have been shown to enhance adherence and improve targeted diet-related outcomes (24). Self-monitoring involves the client keeping a record of thoughts, emotions, dietary behaviours, and health measurements, such as blood pressure. The patients can review this record for

Table 2. Reported BP at baseline and study completion

\begin{tabular}{|c|c|c|c|c|c|c|c|c|}
\hline \multirow{2}{*}{ Items } & \multicolumn{2}{|c|}{ Intervention Group $(n=64)$} & \multicolumn{2}{|c|}{ Control Group $(n=61)$} & \multicolumn{2}{|c|}{$p$-Value } & \multicolumn{2}{|c|}{ Difference $(95 \% \mathrm{CI})$} \\
\hline & SBP & DBP & SBP & DBP & SBP & DBP & SBP & DBP \\
\hline Baseline BP & 135.2 & 81.6 & 131.8 & 76.6 & 0.166 & 0.003 & 3.4 & 5.0 \\
\hline $\mathrm{mmHg}(\mathrm{SD})$ & (13.1) & $(8.7)$ & (14.1) & $(9.6)$ & & & $(-1.4$ to 8.2$)$ & (1.7 to 8.2 ) \\
\hline 12 Week BP & 134.2 & 79.4 & 136.7 & 77.5 & 0.242 & 0.283 & -2.5 & 1.9 \\
\hline $\mathrm{mmHg}(\mathrm{SD})$ & $(10.4)$ & $(10.1)$ & (13.8) & $(8.8)$ & & & $(-6.9$ to 1.8$)$ & $(-1.6$ to 5.3$)$ \\
\hline Difference & -1.1 & -2.2 & 4.9 & 1.2 & 0.021 & 0.026 & -6.0 & -3.4 \\
\hline $\mathrm{mmHg}(\mathrm{SD})$ & (13.0) & $(8.5)$ & $(15.5)$ & (8.6) & & & $(-11.0$ to -0.9$)$ & $(-6.5$ to -0.2$)$ \\
\hline
\end{tabular}

$\mathrm{BP}$, blood pressure.

Table 3. Mixed-effect model for repeated measures (MMRM) analysis of SBP and DBP

\begin{tabular}{lcc}
\hline Dependent Variable (Intervention: $n=64$, Control: $n=61)$ & Estimate difference & $p$-Value \\
\hline Morning SBP (mmHg) & -4.5 & -8.5 to -0.6 \\
Ar(1)* & & 0.024 \\
Morning DBP (mmHg) & -1.8 & -4.4 to 0.8 \\
Ar(1)* & 0.169 \\
\hline
\end{tabular}

SBP: systolic blood pressure, DBP: diastolic blood pressure, $\operatorname{Ar}(1)^{*}$ : auto regressive(1).

Table 4. Clinical and humanistic parameters of patients at baseline and study completion

\begin{tabular}{|c|c|c|c|c|c|c|c|}
\hline \multirow{2}{*}{ Items } & \multicolumn{3}{|c|}{ Intervention group $(n=64)$, Mean (SD) } & \multicolumn{3}{|c|}{ Control group $(n=61)$, Mean (SD) } & \multirow{2}{*}{$p$-Value } \\
\hline & Baseline & After & Difference & Baseline & After & Difference & \\
\hline Medication adherence $^{a}$ & $13.3(1.2)$ & $13.6(1.0)$ & $0.3(1.0)$ & $13.6(1.0)$ & $13.7(1.0)$ & $0.1(1.1)$ & 0.398 \\
\hline $\mathrm{WHO}^{-5^{\mathrm{b}}}$ & $13.2(4.4)$ & $13.2(4.2)$ & $-0.1(2.7)$ & $12.9(4.2)$ & $13.6(4.1)$ & $-0.1(3.1)$ & 0.613 \\
\hline EQ-5D ${ }^{c}$ & $0.90(0.14)$ & $0.89(0.14)$ & $-0.01(0.1)$ & $0.92(0.14)$ & $0.88(0.18)$ & $0.00(0.19)$ & 0.797 \\
\hline BMI d & $24.2(3.3)$ & $24.0(2.5)$ & $-0.1(0.5)$ & $24.3(3.6)$ & $24.4(3.5)$ & $0.0(0.6)$ & 0.768 \\
\hline Steps/day & $6092(3134)$ & $5201(3322)$ & $-993(2064)$ & $5099(2684)$ & $4587(2772)$ & $-614(1819)$ & 0.502 \\
\hline IPAQ $^{\mathrm{e}}$ & $266(228)$ & $243(225)$ & $-23(105)$ & $245(235)$ & $385(270)$ & $119(191)$ & 0.016 \\
\hline Importance ${ }^{\mathrm{f}}$ & $27.0(3.4)$ & $28.3(4.1)$ & $1.23(3.1)$ & $27.1(4.2)$ & $28.1(2.6)$ & $0.96(2.7)$ & 0.636 \\
\hline Confidence $^{\mathrm{g}}$ & $23.5(4.1)$ & $24.2(4.0)$ & $0.6(3.0)$ & $23.9(4.5)$ & $24.8(3.8)$ & $0.87(3.0)$ & 0.690 \\
\hline Salt-intake Score ${ }^{\mathrm{h}}$ & $15.6(3.3)$ & $15.6(3.8)$ & $0.0(2.6)$ & $14.3(2.5)$ & $14.6(3.3)$ & $0.4(2.5)$ & 0.476 \\
\hline Knowledge $^{\mathrm{i}}$ & $5.6(2.5)$ & $6.5(2.1)$ & $0.9(2.1)$ & $5.6(1.9)$ & $6.6(2.2)$ & $1.0(1.8)$ & 0.768 \\
\hline
\end{tabular}

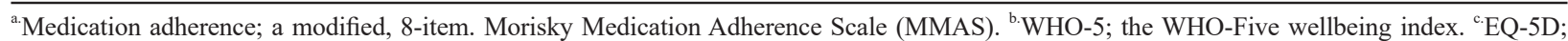
standardized instrument for use as a measure of health outcome. ${ }^{\mathrm{d}} \mathrm{BMI}$; body mass index. ${ }^{\mathrm{e}}$ IPAQ; International Physical Activity Questionnaire. ${ }^{\mathrm{f}}$ ${ }^{\mathrm{g}}$ Importance/Confidence; Pharmacists' importance and confidence scores of diabetes care (out of 10). ${ }^{\text {h }}$ Salt-intake Score; Intake frequency of the following foods, ranging from "everyday" to "not at all". 1) Salted fish, 2) Dried fish, 3) Processed fish meat, 4) Ham and sausage, 5) Pickles, 6) Noodles, 7) Miso soup. ${ }^{i}$ Knowledge of healthy lifestyle choices, measured by 10 true or false questions. 
triggers and patterns, which can then be used to assist with problem solving and goal setting (25). Although community pharmacists have previously conducted randomized controlled trials on lifestyle intervention that were based on motivational interviewing such as cognitive behavioural therapy, all of these trials were focused on improving medication adherence rather than mediating lifestyle changes (26). In our study, however, there was not a significant difference in adherence between the two groups. Thus, the change in BP in our study may reflect the effects of the pharmacists' support for patients.

Previous community pharmacy-based studies measured BP at pharmacies or clinics (12-14). In our study, we were able to analyse home BP measurement data collected during an 84-day period. The mixedeffect model repeated measure (MMRM) results suggest that the intergroup difference in SBP was significant, although the sample of data for analysis was small (125 patients). Therefore, a pharmacist-based intervention could likely promote improvements in BP.

We did not observe changes in physical activities, BMI, and lifestyle choices. The observed intergroup difference in BP might be attributable to the pharmacists' recommendations to the intervention participants regarding reduced sodium intake. Japanese people, especially those who are older, have high levels of sodium intake ( $>10 \mathrm{~g}$ salt per day) $(5,7)$. Because the participants in the current study were older and were likely to have high salt intake, they might have benefitted from the present intervention. However, a 24-hour urine collection analysis was needed to confirm this hypothesis, and this was not possible for this trial due to the practice setting.

It would be expected that the difference in BP observed in this study, about $6 \mathrm{mmHg}$, would be additive to other pharmacist interventions, for which there is substantial high quality evidence, such as recommending medication changes to the physician (26), or independent prescribing by pharmacists (27). As such, adding a brief motivational interview-based intervention could greatly enhance pharmacists' other clinical care.

Our study had some limitations of note. First, approximately one-quarter of the baseline data ( $n=$ 34) were missing. To address this, we used the average values from the first week of home readings, but these were likely lower than in-pharmacy measures, which may have decreased the differences between the two groups. Second, this was an open-label trial and all data were obtained from patients. Therefore, patients in both groups might have reported "desirable" data - an effect that might be greater in the Intervention group. Thirdly, though the difference between both arms was $-6.0 \mathrm{mmHg}$, the change was mainly due to an increase in BP in the Control group $(+4.9 \mathrm{mmHg})$ rather than a reduction in the Intervention group $(-1.1 \mathrm{mmHg})$. This study was carried out from autumn to winter (112/125 patients from September to December) in Japan, where a $15^{\circ} \mathrm{C}$ drop in temperature occurs between September $\left(23.2^{\circ} \mathrm{C}\right)$ and December $\left(6.7^{\circ} \mathrm{C}\right)(28) . \mathrm{A} 1^{\circ} \mathrm{C}$ decrease in the mean outdoor temperature is associated with a rise of $0.41-0.43 \mathrm{mmHg}$ in SBP in Japan $(29,30)$. Thus, in this study, the pharmacists' intervention may have prevented the increase in BP caused by the drop in outdoor temperature, rather than having failed to cause an overall reduction in $\mathrm{BP}$.

We attempted to reduce the risk of sampling bias by only including patients who adhered sufficiently to the strict status recording protocol during a 2-week run-in period. In other words, the present findings were based on what were likely already motivated patients. Another approach is needed to target less motivated patients in a real-world setting. However, most hypertensive patients in Japan have BP monitors in their homes. Accordingly, the results suggest that many of these patients could improve their BP through self-measures if they were educated and supported by pharmacists.

In conclusion, the COMPASS-BP study demonstrated that brief motivational advice given by community pharmacists could potentially improve the control of BP, particularly morning SBP measured at home, among hypertensive patients. Our findings indicate the benefits of implementing a pharmacist-provided lifestyle advice program for patients with chronic disease.

\section{Acknowledgements}

This work was supported by a KAKENHI Grant-in-Aid for Scientific Research (C 24590643), (C 16K08434). We thank the generous collaboration of pharmaceutical associations (Odawara, Shimonoseki, HachimannGamou Nagasaki), pharmacists' groups (Three Star Pharmacists, Kumamoto, Osaka, Nara, Kanazawa) and Hanshin Dispensing Pharmacy Co., Ltd.. We would like to thank Dr. Yazid N. Al Hamarneh and Mr. Eric Duong of University of Alberta, and Mr. Shin Sukino of Kyoto Medical Center for supporting this research.

\section{References}

1. Kario K, Ishikawa J, Pickering TG, Hoshide S, Eguchi K, Morinari M, Hoshide Y, Kuroda T, Shimada K. Morning hypertension: The strongest independent risk factor for stroke in elderly hypertensive patients. Hypertens Res. 2006; 29:581-587.

2. Asayama K, Ohkubo T, Kikuya M, Obara T, Metoki $\mathrm{H}$, Inoue R, Hara A, Hirose T, Hoshi H, Hashimoto J, Totsune K, Satoh H, Imai Y. Prediction of stroke by home "morning" versus "evening" blood pressure values: The Ohasama study. Hypertension. 2006; 48:737-743.

3. World Health Organization. A global brief on hypertension: Silent killer, global public health crisis. http://apps.who.int/iris/bitstream/10665/79059/1/WHO DCO_WHD_2013.2_eng.pdf?ua=1 (accessed October 8, 2017)

4. Miura K, Nagai M, Ohkubo T. Epidemiology of 
hypertension in Japan: Where are we now? Circ J. 2013; 77:2226-2231.

5. Shimamoto K, Ando K, Fujita T, et al. The Japanese Society of Hypertension Guidelines for the Management of Hypertension (JSH 2014). Hypertens Res. 2014; 37:253-390.

6. Cappuccio FP, Kerry SM, Forbes L, Donald A. Blood pressure control by home monitoring: Meta-analysis of randomised trials. BMJ. 2004; 329:145.

7. Japan Ministry of Health, Labour and Welfare. National Health and Nutrition Survey 2010. http://www.mhlw.go.jp/ bunya/kenkou/eiyou/dl/h22-houkoku-09.pdf (accessed October 8, 2017).

8. Neaton JD, Grimm RH Jr, Prineas RJ, et al. Treatment of Mild Hypertension Study. Final results. Treatment of Mild Hypertension Study Research Group. JAMA. 1993; 270:713-724.

9. Uechi K, Asakura K, Masayasu S, Sasaki S. Withincountry variation of salt intake assessed via urinary excretion in Japan: A multilevel analysis in all 47 prefectures. Hypertens Res. 2017; 40:598-605.

10. Sacks FM, Svetkey LP, Vollmer WM, Appel LJ, Bray GA, Harsha D, Obarzanek E, Conlin PR, Miller ER 3rd, Simons-Morton DG, Karanja N, Lin PH; DASH-Sodium Collaborative Research Group. Effects on blood pressure of reduced dietary sodium and the Dietary Approaches to Stop Hypertension (DASH) diet. DASH-Sodium Collaborative Research Group. N Engl J Med. 2001; 344:3-10.

11. Dickinson HO, Mason JM, Nicolson DJ, Campbell F, Beyer FR, Cook JV, Williams B, Ford GA. Lifestyle interventions to reduce raised blood pressure: A systematic review of randomized controlled trials. J Hypertens. 2006; 24:215-233.

12. Aguiar PM, Balisa-Rocha BJ, Brito Gde C, da Silva WB, Machado M, Lyra DP Jr. Pharmaceutical care in hypertensive patients: A systematic literature review. Res Social Adm Pharm. 2012; 8:383-396.

13. Cheema E., Sutcliffe P, Singer DR. The impact of interventions by pharmacists in community pharmacies on control of hypertension: A systematic review and meta-analysis of randomized controlled trials. Br J Clin Pharmacol. 2014; 78:1238-1247.

14. Santschi V, Chiolero A, Colosimo AL, Platt RW, Taffé P, Burnier M, Burnand B, Paradis G. Improving blood pressure control through pharmacist interventions: A meta-analysis of randomized controlled trials. J Am Heart Assoc. 2014; 3:e00718.

15. Topouchian J, Agnoletti D, Blacher J, Youssef A, Ibanez I, Khabouth J, Khawaja S, Beaino L, Asmar R. Validation of four automatic devices for self-measurement of blood pressure according to the international protocol of the European Society of Hypertension. Vasc Health Risk Manag. 2011; 7:709-717.

16. Morisky DE, Ang A, Krousel-Wood M, Ward HJ. Predictive validity of a medication adherence measure in an outpatient setting. J Clin Hypertens. 2008; 10:348-354.

17. Murase N, Katsumura T, Ueda C, Inoue S, Shimomitsu T. Validity and reliability of Japanese version of International
Physical Activity Questionnaire. Journal of Health and Welfare Statistics. 2002; 49:1-9. (in Japanese)

18. Craig CL, Marshall AL, Sjöström M, Bauman AE, Booth ML, Ainsworth BE, Pratt M, Ekelund U, Yngve A, Sallis JF, Oja P. International physical activity questionnaire: 12-country reliability and validity. Med Sci Sports Exerc. 2003; 35:1381-1395.

19. Arakawa K, Matsushita Y, Hrada J, Iwashita M. Obesity and diabetes. 2010; 9:870-872. (in Japanese)

20. Ikeda S, Shiroiwa T, Igarashi A, Noto S, Fukuda T, Saito S, Shimozuma K. Developing a Japanese version of the EQ-5D-5L value set. Journal of the National Institute of Public Health. 2015; 64:47-55. (in Japanese)

21. Machin D, Campbell MJ, Tan SB, Tan SH. Sample size tables for clinical studies, 3rd Edition. Wiley-Black well. 2008.

22. Mallinckrodt $\mathrm{CH}$, Clark WS, David SR. Accounting for dropout bias using mixed-effects models. J Biopharm Stat. 2001; 11:9-21.

23. Miller WR, Rollnick S. Motivational interviewing: Preparing people for change 2nd edition. Guilford Press, New York, USA, 2002.

24. Spahn JM, Reeves RS, Keim KS, Laquatra I, Kellogg M, Jortberg B, Clark NA. State of the evidence regarding behavior change theories and strategies in nutrition counselling to facilitate health and food behavior change. J Am Diet Assoc. 2010; 110:879-891.

25. Hedegaard U, Hallas J, Ravn-Nielsen LV, Kjeldsen LJ. Process- and patient-reported outcomes of a multifaceted medication adherence intervention for hypertensive patients in secondary care. Res Social Adm Pharm. 2016; 12:302-318.

26. Santschi V, Rodondi N, Bugnon O, Burnier M. Impact of electronic monitoring of drug adherence on blood pressure control in primary care: A cluster 12-month randomised controlled study. Eur J Intern Med. 2008; 19:427-434.

27. Tsuyuki RT, Houle SK, Charrois TL, Kolber MR, Rosenthal MM, Lewanczuk R, Campbell NR, Cooney D, McAlister FA; RxACTION Investigators. Randomized trial of the effect of pharmacist prescribing on improving blood pressure in the community: The Alberta Clinical Trial in Optimizing Hypertension (RxACTION). Circulation. 2015; 132:93-100.

28. Japan Meteorological Agency. http://www.jma.go.jp/jma/ indexe.html (accessed October 8, 2017)

29. Kimura T, Senda S, Masugata H, Yamagami A, Okuyama H, Kohno T, Hirao T, Fukunaga M, Okada H, Goda F. Seasonal blood pressure variation and its relationship to environmental temperature in healthy elderly Japanese studied by home measurements. Clin Exp Hypertens. 2010; 32:8-12.

30. Iwabu A, Konishi K, Tokutake H, Yamane S, Ohnishi $\mathrm{H}$, Tominaga Y, Kusachi S. Inverse correlation between seasonal changes in home blood pressure and atmospheric temperature in treated-hypertensive patients. Clin Exp Hypertens. 2010; 32:221-226.

(Received October 10, 2017; Revised December 2, 2017; Accepted December 10, 2017) 